\title{
INTERSTELLAR TRAVEL GUIDE CHRONICLES OF A VIOLENT UNIVERSE
}

\author{
MANEL PERUCHO AND José ANTONIO FonT
}

In contrast to observations from our local viewpoint, the universe is extremely violent. Explosions of massive stars, X-ray and gamma-ray binary stars, and active galaxies are related to the presence of compact objects such as neutron stars or black holes. Processes such as matter accretion or the formation and release of relativistic plasma jets are responsible for the generation of large amounts of high-energy radiation, caused by the acceleration of elementary particles, and have a significant impact in their surroundings. Moreover, they are related to the generation of gravitational waves: vibrations in spacetime produced by the acceleration of compact massive objects that produce strong curvatures. These systems are studied within the framework of the general and special relativity theories. In this article, we summarise several of the most well-known astrophysical scenarios, and offer a brief description of what we currently know about them.

Keywords: relativity, black holes, neutron stars, active galaxies, hydrodynamics.

From our corner, we observe the universe as beings who, not having ever left their homeland, look from a distance and judge everything that happens in other places with the same criteria they use to judge that which occurs at home. These processes are not observed objectively. They are seen through the lens of our external conditions. Therein lies the error: we look up without realising that we can only ponder these questions because we exist, we are aware, and have intelligence. We are aware that we exist, and have the intelligence that is necessary for reflection. But we incur an even more serious error when we think we can extrapolate our conditions everywhere in the universe: if we exist and are aware, it is because

\section{«IF WE EXIST AND ARE \\ AWARE, IT IS BECAUSE NO CATASTROPHIC ASTRONOMICAL EVENT HAS OCCURRED AROUND US}

IN THE LAST FEW MILLION YEARS" the movie September), and if life has developed on our small planet, it is because of, for instance, the absence of nearby persistent or temporary sources of very high-power gamma rays (the most energetic radiation type known). It is also because our star is not sufficiently massive to exhaust its hydrogen stage in only a few million years and then die in a violent explosion.

Interestingly, there is an aspect that relates most astrophysical scenarios to very energetic or violent events: the presence or formation of compact objects. In astrophysics, we consider a massive and (relatively) small object to be compact. They are precisely those in which the Newtonian description of gravity and classical mechanics stop being valid and where we need the geometric description of gravitation published by Einstein in 1915. The field of astrophysics that focuses on studying these objects, as well as the processes involved in their formation and existence, has been known as «relativistic astrophysics» since the 1960s (Shapiro \& Teukolsky, 1983/2007). 

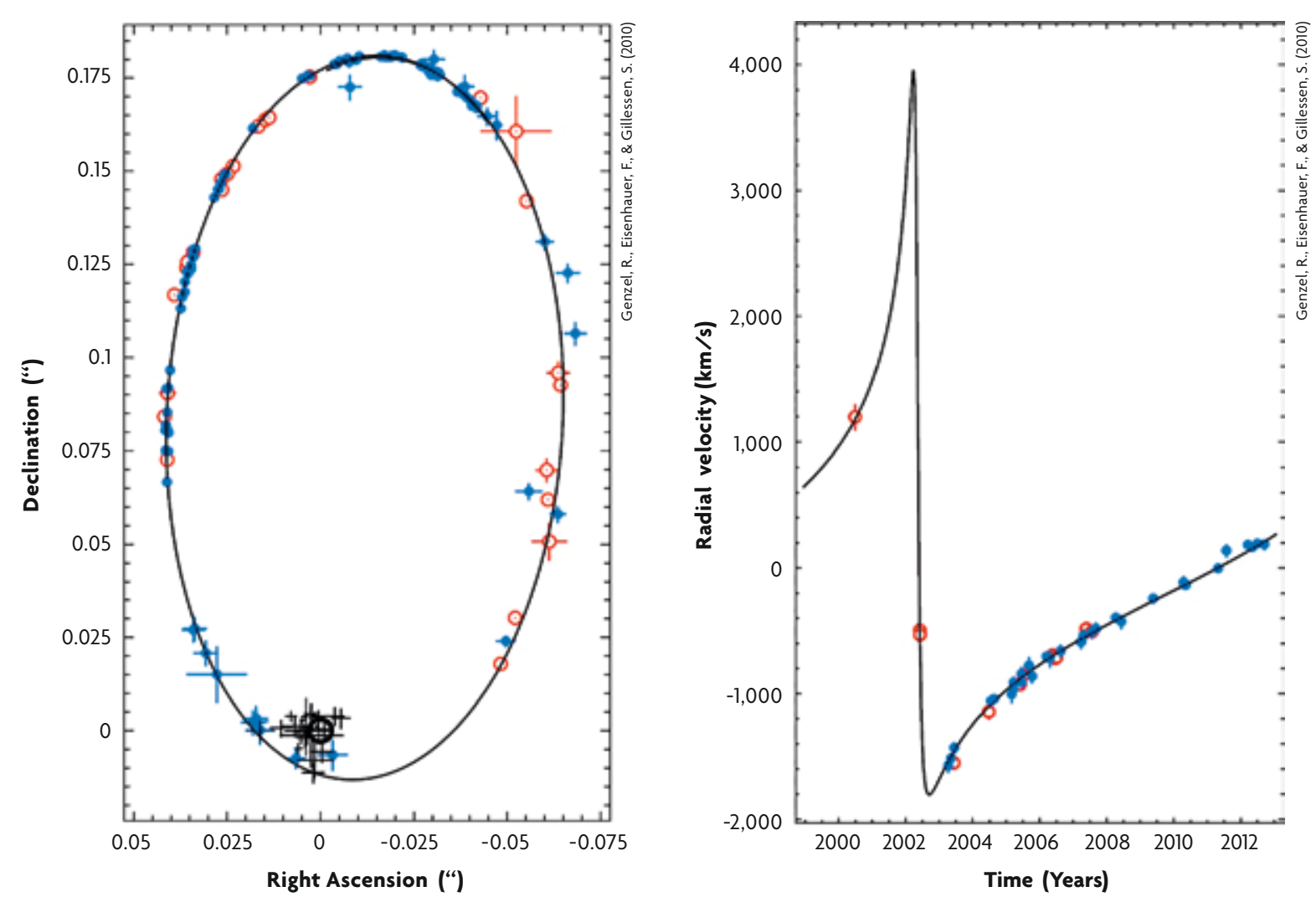

Figure 1. Tracking the stars closest to the centre of our galaxy with infrared telescopes (VLT and Keck) for two decades allowed us to draw their orbits perfectly, and thus, to calculate the mass of the central object around which they orbit. The picture shows the orbit of the closest star to the supermassive object (on the left) and its radial velocity (on the right). Blue points correspond to VLT measurements, while red points correspond to Keck measurements. The crosses indicate the error bars on the measurement of the location of the object. The location of Sagittarius A* is marked with a black circle. It has been calculated that it has a mass of four million solar masses.

This field grew when astronomy developed beyond the optical spectrum, because that narrow window used by our eyes (and traditional telescopes) to observe the sky does not reveal all the information we receive from the cosmos. In addition, the majority of relativistic astrophysics phenomena are not detected in this band. In these scenarios, particles such as electrons, or their antiparticles, the positrons, are accelerated to speeds close to the speed of light, and can reach a total energy several orders of magnitude higher than their resting energy. The particles that reach these energies release radiation that ranges from radio to gamma rays, depending on the processes by which they radiate. Again, classical mechanics are not useful, and here we need Einstein's 1905 theory of special relativity.

\section{OBSERVATIONAL EVIDENCE: GALACTIC NUCLEI}

These extreme scenarios frequently appear in the presence of compact objects related to the last stages of star progression (white dwarfs, neutron stars, and black holes). At much larger scales, there is evidence pointing to the presence of supermassive black holes in the nuclei of galaxies and quasars (Netzer, 2013). Both white dwarfs and neutron stars have been directly observed in different bands of the electromagnetic spectrum. However, in the case of black holes, direct observation is obviously impossible, because the curvature they produce in spacetime even captures the light. We could say that the inside of a black hole is causally disconnected from the outside: even the surface separating the «inside» and the «outside» is known as «event horizon». Nonetheless, theoretical predictions indicate that there are several indirect ways of detecting them, and high-resolution observations have confirmed this.

For instance, the Doppler effect of the light we receive from moving emitters (similar to the Doppler effect in sound waves) allowed experts to directly calculate the orbital periods of stars in a binary system (or their brightness fluctuations), thus enabling them to measure the mass of the invisible object that 
accompanies them. In many of these systems, these compact objects (a neutron star or a black hole) can attract gas from the outermost layers of its companion star when it starts growing (in the last stages of its evolution) and these layers get close enough to the object. In this process of star cannibalization, highenergy radiation is produced in the form of X-rays or, in some cases, gamma rays.

Regarding the black holes at galaxy centres, theory states that in space regions which are curved by a massive object, there is a «time dilation» with respect to the non-curved surrounding areas. This «dilation» produces a redshift in electromagnetic radiation. As a result, it reaches the distant observer in a lower frequency (due to this time dilation) and with a higher, redder, wavelength. This effect has been observed at the centre of active galaxies, in emission lines (the iron K-alpha line) that are wider towards the red end than towards the blue one, and they clearly indicate a gravitational redshift.

In the case of the centre of the Milky Way, we have even stronger evidence: the study of the orbits of different stars around a single point has enabled a Keplerian description of their orbit (like that of a planet's around a star) around a compact and invisible object of roughly four million solar masses (Figure 1), i.e., a supermassive black hole, known as Sagittarius A*. In spite of this, new detectors are being developed at the moment and it is hoped they will offer a clearer picture of the immediate surroundings of this entity, which is assumed to be a black hole. Therefore we have, for instance, the Event Horizon Telescope (EHT), a millimetre-accurate interferometric wavelength observatory ${ }^{1}$ comprising radio telescopes in Chile, Hawaii, Arizona, Spain, France, and Antarctica. Or a new experiment that will take place at the Very Large Telescope (VLT), a group of optical and infrared telescopes located in Chile and based on the interferometry of these spectral bands. These two observatories, together with future instruments such as the Square Kilometer Array, are expected to soon

\footnotetext{
1 Interferometry is a technique consisting in the observation of an object with several separated detectors simultaneously, in order to obtain results equivalent to those of a detector as large as the distance between the individual elements. In the case of antennae separated by continental distances, this is equivalent to having a telescope thousands of kilometres wide.
}

provide observations of the outline of a black hole, known as its «shadow» (see, for example, Goddi et al., 2016).

One of the keys to indirectly observing a black hole is the curvature it produces in the beams of light that pass by it. Sir Arthur Eddington's 1919 experiment during a solar eclipse proved that this effect, predicted by the theory of general relativity, was correct. In the case of black holes, the extreme curvature of the light emitted in its surroundings, for example in the disk of matter falling towards it, would give it an appearance similar to what we see in Figure 2, close to the image of the fictional black hole Gargantua, popularised by the movie Interstellar. It is possible to observe the black hole at the centre of our galaxy, Sagittarius A*, because it is the closest to us, although we will need very precise observations to describe its shadow. In fact, its angular size in the sky is of around 37 microarcseconds, the equivalent to observing a racquet ball on the Moon from Earth, or a bacterium from $20 \mathrm{~km}$ away.

Despite the fact that all the scenarios we have described are observed in our galaxy, the term «relativistic astrophysics» was not created to refer to the observation of galactic objects. Rather, it was used for the first time in quasar discovery. In 1962, Maarten Schmidt announced the detection of spectral lines of hydrogen in the spectrum of quasar 3C273. These emission lines clearly shifted to red, which when taking Hubble's law into account, indicate that $3 \mathrm{C} 273$ is a faraway, extragalactic source. Soon they deduced that it was a galaxy and that its quasi-stellar appearance (hence the name QSO, Quasi Stellar Object, or quasar for radio sources) was due to the fact that most of the radiation we receive from it is produced in a small region within it. In this case, a supermassive black hole, around a hundred or a thousand times the mass of Sagittarius A* (that is to say, between a hundred thousand and a thousand million solar masses) located at the centre of a galaxy which captures matter from a gas disk around it. The rate of matter falling inwards is around a solar mass (or a large fraction of it) per year. That amount of matter produces energy amounts comparable to the conversion of Earth's mass into energy $\left(\mathrm{E}_{0}=\mathrm{m} \mathrm{c}^{2} \sim 10^{40-41} \mathrm{~J}\right)^{2}$ every second.

\footnotetext{
$21 \mathrm{~J}=1 \mathrm{~kg} \mathrm{~m}^{2} / \mathrm{s}^{2}$, international energy unit.
} 


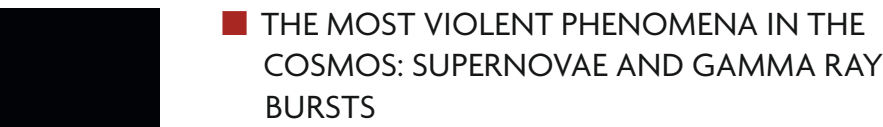

At the end of its thermonuclear stage, stars with an initial mass between 9 and 30 solar masses (a solar mass equivalent to $1,989 \times 10^{30} \mathrm{~kg}$ ) are not capable of producing enough energy to sustain their mass. Thus, the star collapses on itself. This collapse stops when matter reaches densities above those of atomic nuclei $\left(\sim 10^{17} \mathrm{~kg} / \mathrm{m}^{3}\right)$ at which point their collapse instead forms neutron stars. If progenitor stars in this process are sufficiently massive, the collapse might continue and result in the formation of a black hole. In a neutron star, matter is so compressed that a spoonful of it would amount to a mass of 100 million tonnes.

After the sudden stop in the collapse of that central compact object, there is a violent bounce that releases a (gravitational) energy of $10^{46} \mathrm{~J}$, in a shockwave that pushes the outermost layers of the star and produces a supernova explosion. The dynamics of this extreme process can only be studied through its relativistic description and numerical calculations performed by supercomputers; these virtual laboratories allow us to scrutinise scenarios that cannot be reproduced in laboratories (see, for example, Janka, 2012).

As we anticipated, the result of these violent explosions is either the formation of a neutron star or a black hole. The radius of the former is around a hundred thousand times smaller than that of the Sun's (which is $6.96 \times 10^{8} \mathrm{~m}$ ) and therefore has a much higher surface gravitational potential and an escape velocity of about a third of the speed of light. The internal composition and thermodynamic properties of neutron stars remain hidden from us. These aspects are also studied through numerical simulations, since the results obtained by modifying the critical parameters of theoretical models can be compared with observational properties (for instance, the surface oscillations of the stars under study). Moreover, some neutron stars (pulsars) emit quick radiation pulses in radio frequencies due to a combination of high rotation speeds and the presence of intense magnetic fields on their surface. The first of them was observed in 1967 by Jocelyn Bell and Antony Hewish (Hewish, Bell, Pilkington, Scott, \& Collins, 1968). Finally, some neutron stars are observed indirectly as regular $\mathrm{X}$-ray or gamma ray sources in binary star systems, in which one of the components is precisely one of these compact objects.

The collapse of a high-mass star and the subsequent formation of a black hole, or the final moments of the spiral collision of two neutron stars in a binary system where the two stars have gone supernova, produces one of the most energetically violent phenomena in the cosmos: gamma-ray bursts. Depending on their duration, they are classified into two groups: long flashes ( $20 \mathrm{~s}$ ) associated with a «failed» stellar collapse (known as the collapsar model because a neutron star is not formed) and short flashes ( 0.2 s) associated with the collision of two compact objects. Fortunately for life on Earth, these gamma ray bursts are extragalactic events that are only observed at cosmological distances (billions of light years), because if they occurred in regions near our galaxy, they could destroy our atmosphere (Piran \& Jiménez, 2014). The fact that they can be observed at such distances implies that they are an extremely bright phenomenon in which enormous amounts of energy (around $10^{44} \mathrm{~J}$ ) are released in the form of gamma rays; in the time that one of these bursts lasts, the equivalent of one solar mass could be transformed into gamma radiation. This radiation is not emitted isotropically, it is collimated in the direction the particles are propagated. This is a known relativistic effect and is caused because the particles that emit it propagate at close to the speed of light and form a jet, thus, focussing the radiation along a tight beam. Estimates indicate that particles propagate at ultrarelativistic speeds, at more than $0.9999 c$, where $c$ is the speed of light (Gehrels, Ramírez-Ruiz, \& Fox, 2009).

\section{THE MOST FEARSOME MONSTERS}

The German physicist Karl Schwarzschild obtained the first solution to Einstein's equations barely a month after the publication of the theory of general relativity in 1916. His solution provides the distribution of the static gravitational field outside of a massive object with spherical symmetry and in the absence of electrical charges. The solution offers the relevant (and singular) conclusion that for each mass, distributed in the form of a sphere, there is a radius value under which not even light can escape the corresponding surface; that is, it announced the possibility that black holes could exist. Much later, in 1963, the New Zealand mathematician Roy Patrick Kerr obtained a 


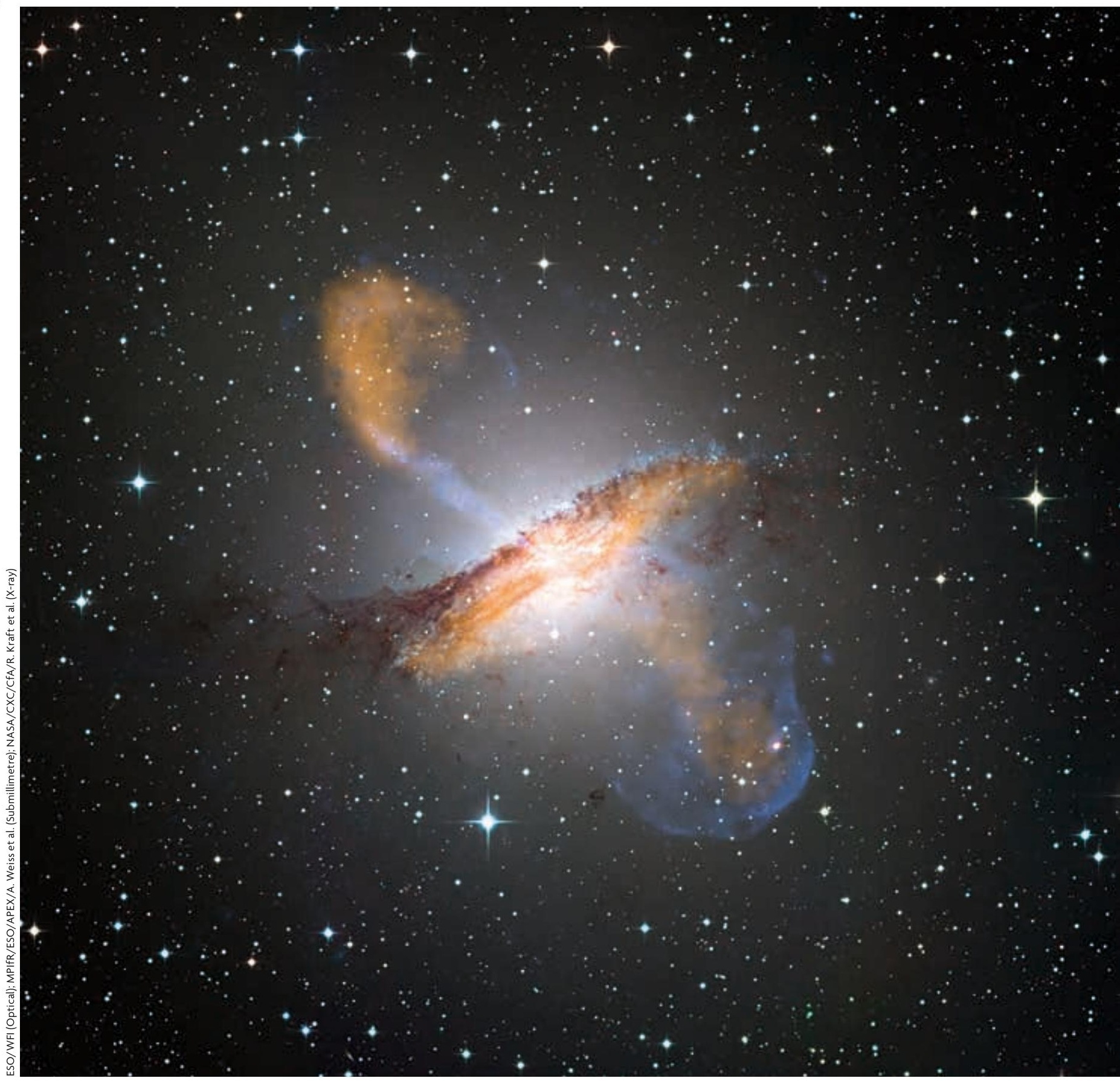

Figure 3. Combination of radio, optical, and X-ray images of the radiogalaxy Centaurus $\mathrm{A}$. Active galaxies contain a supermassive black hole at their centre, surrounded by an accretion disk. The material in the disk generates enormous amounts of energy as it falls towards the central object. In this process, which is the most energetically efficient that we know of, particles can yield between ten and forty percent of their mass in the form of energy. In this way, the central region of such galaxies is much brighter than that of a normal galaxy. In a region of these galaxies perpendicular to the accretion disc, matter and energy jets are created and propagate over great distances at relativistic speeds, affecting their environment and the evolution of their host galaxy.
"THERE IS EVIDENCE POINTING

TO THE PRESENCE OF

SUPERMASSIVE BLACK HOLES» 
new solution adding the possibility of rotating objects to Schwarzschild's solution. These kinds of solutions describe what we know as the metric of a spacetime, because they determine the temporal and spatial relationships between its events. The derivation of the Kerr metric was not only contemporary to M. Schmidt's work with quasars, but it also became the theoretical basis for explaining the generation of energy in these objects.

Indeed, the capture of matter by a Kerr black hole can turn the region surrounding it into an intense source of radiation. This process, known as «accretion», typically occurs as a rotating disk of matter. The viscosity of the disk causes particles to lose energy and to heat it while they fall towards the event horizon of the black hole. In the case of Schwarzschild black holes, the energy released per mass unit in this process is ten times higher than is released by nuclear fusion in star nuclei $\left(\sim 0.07 c^{2}\right)$, and it can reach $40 \%$ of the resting energy of each particle in Kerr black holes. This high efficiency provides a robust theoretical model that explains galactic activity, of which quasars are an archetypal case. Part of this energy is released in the form of radiation in a relatively small region around a supermassive black hole (see Netzer, 2013).

Matter and energy jets are formed in the surrounding areas of supermassive black holes and propagate close to the speed of light along the rotational axis of the central object. These jets travel enormous distances, crossing their progenitor galaxy and altering the subsequent evolution of the galaxy and its environment (Figure 3). The formation of relativistic jets is associated with the extraction of energy from the system: energy from the rotation of the black hole (Blandford-Znajek mechanism; Blandford \& Znajek, 1977) and from the rotation of the disk around it (Blandford-Payne mechanism; Blandford \& Payne, 1982). One of the observational properties of these jets is that some areas of intense radio emission propagate at apparently fasterthan-light speeds. This optical illusion is produced when matter moves at speeds near (but still lower) than that limit, approaching the observer almost directly. This phenomenon has been observed in many radiogalaxies and quasars. Values up to fifty times the speed of light have been measured for this apparent speed.

We still do not know the mechanism behind the formation of high-mass black holes, but current theories posit that they form thanks to the initial collapse of high-mass stars or the collapse of extensive gas clouds present at the centre of protogalaxies in the early stages of the universe. However, there is consensus that their increase in mass over time is due to their fusion with other similar-sized black holes and to gas and star accretion.

In our own galaxy there are reduced versions of this kind of system, related to binary stars where one of the components goes supernova at the end of its thermonuclear stage. When the new neutron star or black hole is formed, the new compact object can
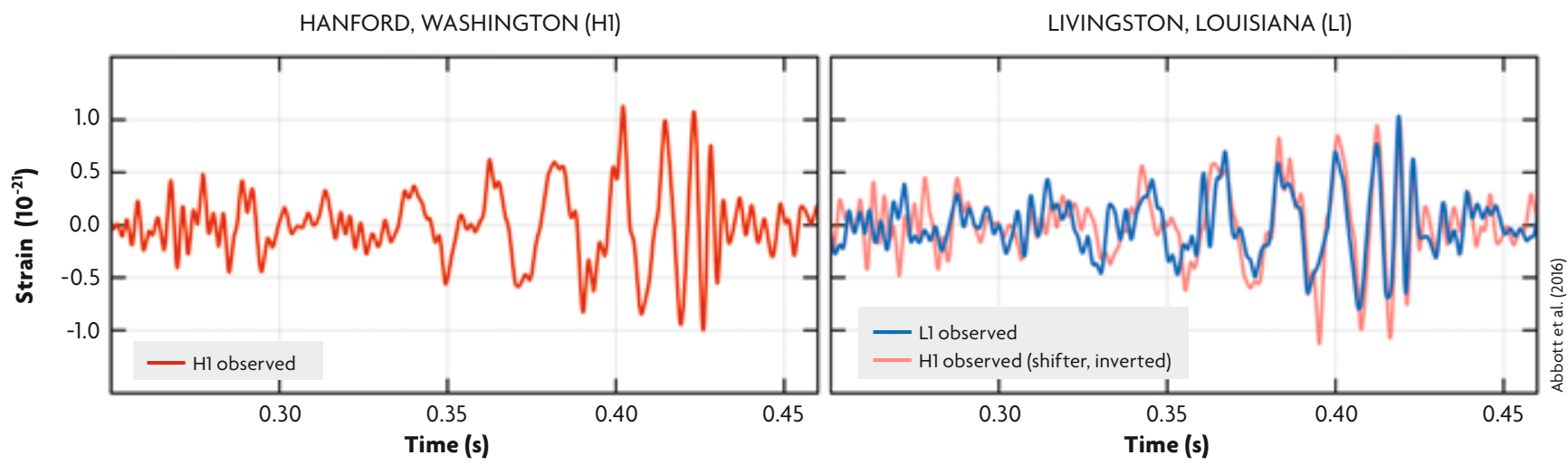

Figure 4. The first gravitational wave signal, GW150914, detected by Advanced LIGO in September 2015. The blue line (L1) shows the signal received by the Livingston detector (Louisiana, USA), and the red line ( $\mathrm{H} 1$ ) shows the signal received in Hanford (Washington, USA) for the same detection. This historical signal opened up a new window onto the universe and started a new revolution in astrophysics and cosmology, comparable to the twentieth-century development of astronomy when scientists started to use the entire electromagnetic spectrum. 


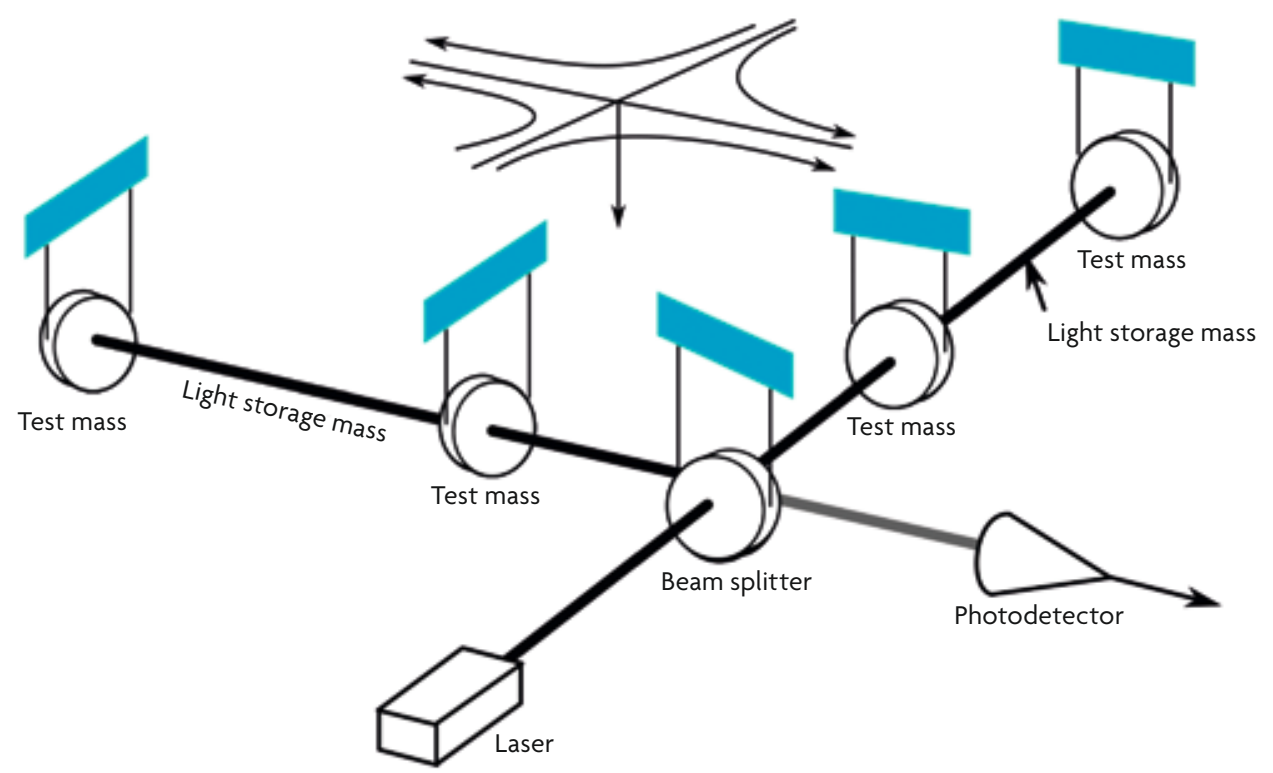

Figure 5. Diagram of a Michelson-Morley interferometer with Fabry-Perot resonant cavities like the ones used by Advanced Virgo and Advanced LIGO. The tidal forces associated with the passage of a gravitational wave infinitesimally modify the distance between test masses, so the laser beam (divided into two at the beam splitter) takes different times to go through each of the branches of the detector. In this way, when the beam is recollimated at the output of the interferometer, the photodetector does not capture a perfectly destructive interference pattern.

attract the external layers of its companion star which is still in its thermonuclear stage. This falling matter generates X-ray emissions and forms relativistic jets that impact the interstellar medium and emit in the radio band. In some cases, jets formed in binary stars are also candidate gamma-radiation sources. In our galaxy, we are aware of twenty of these objects, located thousands of light-years away from us. One of them provided the first direct evidence for the existence of black holes: Cygnus X-1 (Bolton, 1972; Webster \& Murdin, 1972).

\section{A NEW WINDOW TO THE EXTREME UNIVERSE}

Gravitational radiation is also a consequence of the theory of general relativity. This radiation is associated with waves in the curvature of spacetime itself; that is, with oscillations in the fabric of the universe, propagated at light-speed, and carrying information about the astronomical sources that originate them. Gravitational radiation can be produced at detectable levels because of the acceleration of large compact masses, as occurs in collisions of black holes or neutron stars, or in their formation which causes intense distortions in spacetime. In September 2015, experts detected this type of radiation for the first time (detection GW150914, Abbott et al., 2016; Figure 4). From the information obtained, they deduced that the detected gravitational radiation originated in the last instants (milliseconds) of the spiral collision between two Kerr black holes in a binary system.

These objects would have had between 36 and 29 solar masses, respectively, at the time of the collision, and the process released an energy equivalent to 3 solar masses in the form of gravitational radiation. In these types of system, where two compact objects are mutually accelerated, the emission of gravitational waves extracts both energy and angular momentum from the system, so both objects end up falling towards each other, causing an enormous spacetime distortion to occur at the time of the collapse. Thus, each type of collapse produces a different signal, whose theoretical wave forms are built using theoretical approaches for the approach stage (spiral) of the objects, as well as numerical simulations of Einstein's equations for the fusion stage of the two objects. 
The observation of GW150914 provided the first direct evidence of the existence of Kerr black holes, which had been theoretically predicted in 1963. Previously, gravitational waves had only been «detected» indirectly, in connection with the discovery of the binary pulsar PSR 1913+16 in 1974 by Russell Hulse and Joseph Taylor (1975). In this case, the precise measurement of the decrease in the orbital period of the system loss due to the emission of gravitational radiation. However, on 26 December 2015 there was a second (this time direct) detection (GW151226), which marked the start of a new "THE NEW DISCOVERIES WILL PROBABLY INFLUENCE OUR WAY OF UNDERSTANDING THE UNIVERSE»

REFERENCES was related to the energy

Abbott, B. P., Abbott, R., Abbott, T. D., Abernathy, M. R., Acernese, F., Ackley, K., ... Zweizig, J. (2016). Observation of gravitational waves from a binary black hole merger. Physical Review Letters, 116(6), 061102. doi: 10.1103/PhysRevLett.116.061102

Blandford, R. D., \& Payne, D. G. (1982). Hydromagnetic flows from accretion discs and the production of radio jets. Monthly Notices of the Royal Astronomical Society, 199(4), 883-903. doi: 10.1093/mnras/199.4.883 Blandford, R. D., \& Znajek, R. L. (1977). Electromagnetic extraction of energy from Kerr black holes. Monthly Notices of the Royal Astronomical Society, 179(3), 433-456. doi: 10.1093/mnras/179.3.433

Bolton, C. T. (1972). Identification of Cygnus X-1 with HDE 226868. Nature, 235, 271273. doi: $10.1038 / 235271 b 0$

Font, J. A., Sintes, A. M., \& Sopuerta, C. F. (2015). Gravitational waves with the SKA. In M. A. Pérez-Torres (Ed.), The Spanish Square Kilometer Array white book (pp. 29-36). Barcelona: Sociedad Española de Astronomía. Retrieved from https://arxiv. org/abs/1506.03474

Gehrels, N., Ramírez-Ruiz, E., \& Fox., D. B. (2009). Gamma-ray bursts in the Swift era. Annual Review of Astronomy and

of the universe.

The detector with the honour of opening up this new observational window of the most extreme aspects of the universe, with the two first such detections, was the Advanced LIGO. The same as the Advance Virgo and KAGRA instruments, the LIGO detector is a kilometre-scale MichelsonMorley interferometer (Figure 5) that can measure extraordinarily small length-variations. ${ }^{3}$ These distances are comparable to measuring the distance between the Earth and the Sun with the precision of an atom's nucleus. By design, these detectors are sensitive to the typical frequency of the gravitational radiation produced in supernovae or in the collisions of neutron stars or stellar-mass black holes $(\sim 50$ $2000 \mathrm{~Hz}$ ). The future space interferometer eLISA will be sensitive to the frequencies of gravitational radiation emitted in the collision of white dwarfs or in the captures of stellar-mass objects by supermassive black holes ( $\mathrm{mHz}-\mathrm{Hz}$ range). There are also detectors based on the precise measurement of the rotation periods of pulsars (pulsar timing), such as IPTA or the future SKA, which will be sensitive to ultralow frequencies ( $\mathrm{nHz}$ range; see, for example, Font et al., 2015 and references therein). All these detectors open up new spectra and new windows for observing the universe, just as astronomy developed beyond the optical range of the electromagnetic spectrum in the twentieth century. Similarly, new discoveries will probably influence our way of understanding the universe, just as those made during the last century did. (†)

\footnotetext{
3 The effect of tidal forces - spacetime expansions and contractions with an effect similar to Earth's tides - on test masses caused by gravitational waves is minor.
}

Astrophysics, 47, 567-617. doi: 10.1146/annurev.astro.46.060407.145147 Genzel, R., Eisenhauer, F., \& Gillessen, S. (2010). The Galactic Center massive black hole and nuclear star cluster. Reviews of Modern Physics, 82,3121-1395. doi: 10.1103/RevModPhys.82.3121

Goddi, C., Falcke, H., Kramer, M., Rezzolla, L., Brinkerink, T., Bronzwaer, T., ... Zhidenko, A. (2016). BlackHoleCam: fundamental physics of the galactic center. International Journal of Modern Physics D, 1730001. doi: 10.1142/S0218271817300014

Hewish, A., Bell, S. J., Pilkington, J. D. H., Scott, P. F., \& Collins, R. A. (1968). Observation of a rapidly pulsating radio source. Nature, 217 , 709-713. doi: 10.1038/217709a0

Hulse, R. A., \& Taylor, J. H. (1975). Discovery of a pulsar in a binary system. Astrophysical Journal, 195, L51-L53. doi: 10.1086/181708

Janka, H. T. (2012). Explosion mechanisms of core-collapse supernovae. Annual Review of Nuclear and Particle Science, 62, 407-451. doi: 10.1146/annurev-nucl-102711-094901

Mościbrodzka, M., Falcke, H., Shiokawa, H., \& Gammie, C. F. (2014). Observational appearance of inefficient accretion flows and jets in 3D GRMHD simulations: Application to Sagittarius A*. Astronomy \& Astrophysics, 570, A7. doi: 10.1051/0004-6361/201424358

Netzer, H. (2013). The physics and evolution of active galactic nuclei. Cambridge: Cambridge University Press.

Piran, T., \& Jiménez, R. (2014). Possible role of gamma ray bursts on life extinction in the universe. Physical Review Letters, 113(23), 231102. doi: 10.1103/PhysRevLett.113.231102

Shapiro, S. L., \& Teukolsky, S. A. (2007). Black holes, white dwarfs, and neutron stars: The physics of compact objects. New York: Wiley Online Library. (Original work published in 1983). doi: 10.1002/9783527617661

Webster, B. L., \& Murdin, P. (1972). Cygnus X-1 -A spectroscopic binary with a heavy companion? Nature, 235, 37-38. doi: 10.1038/235037a0

Manel Perucho Pla. Tenured professor of Physics at the University of Valencia (Spain). His research focuses on the study of relativistic fluids in different astrophysical scenarios using relativistic hydrodynamics and magnetohydrodynamics numerical simulations. Among the scenarios he studies, are the jets generated in active galactic nuclei or in binary $\mathrm{X}$-ray or gamma-ray stars. He was the principal investigator on different supercomputer calculation projects as well as on an international observation project with the Russian space telescope Radioastron.

José Antonio Font Roda. Tenured professor of Physics at the University of Valencia (Spain). His field of research is relativistic astrophysics and in particular, he studies neutron stars, black holes, and gravitational radiation using computational tools such as relativistic hydrodynamics or magnetohydrodynamics and numerical relativity. He was the president of the Spanish Society of Gravitation and Relativity and now coordinates the Valencia group for the Virgo collaboration and directs the Department of Astronomy and Astrophysics in the University of Valencia. 